\title{
Applications of Picard and Magnus expansions to the Rabi model
}

\author{
Fabrizio Angaroni, ${ }^{1,2}$ Giuliano Benenti, ${ }^{1,2,3}$ and Giuliano Strini ${ }^{4}$ \\ ${ }^{1}$ Center for Nonlinear and Complex Systems, Dipartimento di Scienza e Alta Tecnologia, \\ Università degli Studi dell'Insubria, via Valleggio 11, 22100 Como, Italy \\ ${ }^{2}$ Istituto Nazionale di Fisica Nucleare, Sezione di Milano, via Celoria 16, 20133 Milano, Italy \\ ${ }^{3}$ NEST, Istituto Nanoscienze-CNR, 56126 Pisa, Italy \\ ${ }^{4}$ Department of Physics, University of Milan, via Celoria 16, 20133 Milano, Italy
}

\begin{abstract}
We apply the Picard and Magnus expansions to both the semiclassical and the quantum Rabi model, with a switchable matter-field coupling. The case of the quantum Rabi model ia a paradigmatic example of finite-time quantum electrodynamics (QED), and in this case we build an intuitive diagrammatic representation of the Picard series. In particular, we show that regular oscillations in the mean number of photons, ascribed to the dynamical Casimir effect (DCE) for the the generation of photons and to the anti-DCE for their destruction, take place at twice the resonator frequency $\omega$. Such oscillations, which are a clear dynamical "smoking gun" of the ultrastrong coupling regime, can be predicted by first-order Picard expansion. We also show that the Magnus expansion can be used, through concatenation, as an efficient numerical integrator for both the semiclassical and the quantum Rabi model. In the first case, we find distinctive features in the Fourier spectrum of motion, with a single peak at the Rabi frequency $\Omega$ and doublets at frequencies $2 n \omega \pm \Omega$, with $n$ positive integer. We explain these doublets, which are a feature beyond the rotating wave approximation (RWA), on the basis of the Picard series.
\end{abstract}

\section{INTRODUCTION}

Ultrastrong coupling between artificial atoms and electromagnetic cavity modes is achieved when the coupling strength $\lambda$ becomes comparable to, or even exceeds the resonator frequency $\omega$. Such regime, which is nowadays experimentally addressed in circuit QED [1 [5], is of interest both for the development of quantum technologies and for fundamental physics. Indeed, strong matter-field coupling is preliminary to the implementation of fast quantum protocols. On the other hand, in the ultrastrong coupling regime strongly correlated matter-light states emerge [4, [5].

A prominent phenomenon in ultrastrong matter-field coupling is the dynamical Casimir effect, that is, the generation of photons from the vacuum due to timedependent boundary conditions or, more generally, as a consequence of the nonadiabatic change of some parameters of a system [6] (this latter case is usually refereed to as parametric DCE [7]). DCE has been discussed in several contexts, for instance in Bose-Einstein condensates [9, in excition-polariton condensates [10, for multipartite entanglement generation in cavity networks 11, in relation to several forms of quantum correlations [1214, in the generation of exotic field states [15], for quantum communication protocols [16], in quantum thermodynamics [17; the DCE can also be amplified via optimal control techniques [18. Moreover, pioneering experimental demonstrations of the DCE have been reported in superconducting circuit QED [19, 20].

In contrast with standard QED, here we consider a single (cavity) mode rather than an infinite number of modes. Moreover, the quantization volume (of the cavity) is fixed and the limit of infinite volume is not taken at the end. Finally, the interaction is not switched on and off adiabatically, but we rather focus on transient phenomena associated with the nonadiabatic switching of the matter-field coupling. That is, we are considering finite-time QED, a problem barely considered in the literature [21].

The quantum Rabi model [22, 23], which describes the dipolar light-matter coupling, with the addition of a switchable coupling, is the ideal testing ground to explore finite-time QED in the ultrastrong coupling regime.

In this paper, we examine applications of the the Picard and Magnus expansions to both the semiclassical and the quantum Rabi model, with a time-dependent coupling. While the dynamics of these models can be addressed numerically via a Runge-Kutta integration of the equations of motion, perturbative methods can shed light on the physical mechanisms and elementary processes which govern the dynamics. We first investigate the Picard series, which allows an intuitive diagrammatic representation. Such series, truncated to low orders, provides a rather accurate description only for short interaction times (and not too strong coupling). In particular, we show that regular oscillations in the mean number of photons, can be ascribed to the coherent generation (DCE) and destruction (anti-DCE [24, 25]) of photons. Such oscillations take place at a frequency $2 \omega$ that can be predicted by first-order Picard expansion, and are a clear dynamical "smoking gun" of the ultrastrong coupling regime. We then examine the Magnus expansion, and show that through concatenation it can be used as an efficient numerical integrator. In particular, we study the Fourier spectrum of motion for the semiclassical Rabi model and show that it has a characteristic structure, with a single peak at the Rabi frequency $\Omega$ and doublets at frequencies $2 n \omega \pm \Omega$, with $n=1,2,3, \ldots$. The doublets, which are a feature beyond the RWA, are explained on the basis of the Picard series. Finally, we discuss analogies between the semiclassical Rabi model 
and the Mathieu equation.

\section{THE FINITE-TIME RABI MODEL}

We consider both the semiclassical and the quantum finite-time Rabi models, describing the interaction of a two-level atom (qubit) with the electromagnetic field [22]. In both cases, the Hamiltonian

$$
H(t)=H_{0}+H_{I}(t),
$$

where $H_{0}$ refers to the free evolution for the qubit and the field, and $H_{I}(t)$ describes a time-modulated qubit-field coupling, which extends over a finite time $0 \leq t \leq \tau$.

In the semiclassical Rabi model, which describes within the dipole approximation, the interaction between the qubit and a classical monochromatic field, (hereafter we set the reduced Planck's constant $\hbar=1)$,

$$
\begin{gathered}
H_{0}=-\frac{1}{2} \omega_{q} \sigma_{z}, \\
H_{I}(t)=f(t)[2 \Omega \cos (\omega t+\phi)] \sigma_{x},
\end{gathered}
$$

where $\omega_{q}$ and $\omega$ are the qubit and field frequency, respectively, $\Omega$ is the (Rabi) frequency of the field-induced oscillations between the two levels $|g\rangle$ and $|e\rangle$, the Pauli matrices $\sigma_{k}(k=x, y, z)$ are written in the $\{|g\rangle,|e\rangle\}$ basis, and the function $f(t)$ modulates the qubit-field coupling. Hereafter, for simplicity's sake we shall assume the phase $\phi=0$, the resonant case $\omega_{q}=\omega$, and a sudden switch on/off of the coupling: $f(t)=1$ for $0 \leq t \leq \tau, f(t)=0$ othertwise.

In the case of the quantum Rabi model, which describes the interaction between the qubit and a single mode of the quantized field,

$$
\begin{gathered}
H_{0}=-\frac{1}{2} \omega_{q} \sigma_{z}+\omega\left(a^{\dagger} a+\frac{1}{2}\right), \\
H_{I}(t)=f(t)\left[\lambda \sigma_{+}\left(a^{\dagger}+a\right)+\lambda^{\star} \sigma_{-}\left(a^{\dagger}+a\right)\right],
\end{gathered}
$$

where $\sigma_{ \pm}=\frac{1}{2}\left(\sigma_{x} \mp i \sigma_{y}\right)$ are the raising and lowering operators for the qubit (so that $\sigma_{+}=|e\rangle\langle g|$ and $\left.\sigma_{-}=|g\rangle\langle e|\right): \sigma_{+}|g\rangle=|e\rangle, \sigma_{+}|e\rangle=0, \sigma_{-}|g\rangle=0$, $\sigma_{-}|e\rangle=|g\rangle$. The operators $a^{\dagger}$ and $a$ for the field create and annihilate a photon: $a^{\dagger}|n\rangle=\sqrt{n+1}|n+1\rangle$, $a|n\rangle=\sqrt{n}|n-1\rangle,|n\rangle$ being the Fock state with $n$ photons. For the sake of simplicity, from now on we consider a real coupling strength, $\lambda \in \mathbb{R}, \omega_{q}=\omega$ and a timedependent modulation set as above for the semiclassical model.

The rotating wave approximation (valid for $\lambda \rightarrow 0$ ) is obtained neglecting the term $\sigma_{+} a^{\dagger}$, which simultaneously excites the qubit and creates a photon, and $\sigma_{-} a$, which de-excites the qubit and annihilates a photon. In this limit, the Hamiltonian (3) reduces to the JaynesCummings Hamiltonian 22] with a time-dependent modulation. In the RWA the swapping time needed to transfer an excitation from the qubit to the field or vice versa $(|e\rangle|0\rangle \leftrightarrow|g\rangle|1\rangle)$ is $\tau_{s}=\pi / 2 \lambda$, and no DCE is possible since the total number of excitations in the system is conserved. Within RWA, the (Rabi) frequency of the Rabi oscillations between the states $|e\rangle|n-1\rangle$ and $|g\rangle|n\rangle$ is $\Omega_{n}=\lambda \sqrt{n}$.

In the interaction picture, the Hamiltonian reads $\tilde{H}_{I}(t)=U^{\dagger}(t) H_{I}(t) U(t)$, where $U(t)=e^{-i H_{0} t}$. From now on we shall omit tildes and always refer to the interaction picture. For the semiclassical Rabi model,

$$
H_{I}(t)=\Omega f(t)\left[\left(1+e^{-2 i \omega t}\right) \sigma_{-}+\left(1+e^{2 i \omega t}\right) \sigma_{+}\right],
$$

while in the quantum Rabi model

$$
H_{I}(t)=\lambda f(t)\left[\sigma_{-} a e^{-2 i \omega t}+\sigma_{+} a+\sigma_{-} a^{\dagger}+\sigma_{+} a^{\dagger} e^{2 i \omega t}\right] .
$$

In both cases, the RWA is recovered if we neglect the counter-rotating terms at frequency $2 \omega$.

\section{PICARD SERIES}

The solution to the time-dependent Schrödinger equation $i|\dot{\psi}(t)\rangle=H_{I}(t)|\psi(t)\rangle$ can be approximated by the Picard iterative process. We start by writing the integral associated equation

$$
|\psi(t)\rangle=|\psi(0)\rangle-i \int_{0}^{t} H_{I}\left(t^{\prime}\right)\left|\psi\left(t^{\prime}\right)\right\rangle d t^{\prime} .
$$

Iterating the process we obtain

$$
\begin{gathered}
|\psi(t)\rangle=|\psi(0)\rangle-i \int_{0}^{t^{\prime}} H_{I}\left(t^{\prime}\right)[|\psi(0)\rangle \\
\left.-i \int_{0}^{t^{\prime}} H_{I}\left(t^{\prime \prime}\right)\left|\psi\left(t^{\prime \prime}\right)\right\rangle d t^{\prime \prime}\right] d t^{\prime}
\end{gathered}
$$

and so on. Hence we can write

$$
|\psi(t)\rangle=\sum_{k=0}^{\infty}\left|\psi^{(n)}(t)\right\rangle,
$$

with the zeroth-order approximation $\left|\psi^{(0)}(t)\right\rangle=|\psi(0)\rangle$, the first-order correction

$$
\left|\psi^{(1)}(t)\right\rangle=-i \int_{0}^{t} H_{I}\left(t^{\prime}\right)\left|\psi^{(0)}\left(t^{\prime}\right)\right\rangle d t^{\prime},
$$

and so on, with the $n$-th-order correction given by

$$
\left|\psi^{(n)}(t)\right\rangle=-i \int_{0}^{t} H_{I}\left(t^{\prime}\right)\left|\psi^{(n-1)}\left(t^{\prime}\right)\right\rangle d t^{\prime} .
$$

\section{A. Semiclassical Rabi model}

We expand the state vector in the $\{|g\rangle,|e\rangle\}$ basis for the qubit: $|\psi(t)\rangle=C_{g}(t)|g\rangle+C_{e}(t)|e\rangle$. For concreteness, we consider the initial state $|\psi(0)\rangle=|g\rangle$ (however, the 
considerations of this subsection would not change for a different initial state).

It is instructive to consider first the RWA limit, in which we easily obtain the exact solution to the Schrödinger equation, $|\psi(t)\rangle=\cos (\Omega t)|g\rangle-i \sin (\Omega t)|e\rangle$, corresponding to Rabi oscillations between the two states $|g\rangle$ and $|e\rangle$. In this case, the $n$-th order Picard expansion of $|\psi(t)\rangle$ coincides with the result obtained from the $n$-th order Taylor expansion of the exact coefficients $C_{g}(t)=\cos (\Omega t)$ and $C_{e}(t)=-i \sin (\Omega t)$ :

$$
\begin{gathered}
\left|\psi^{(0)}(t)\right\rangle=|g\rangle,\left|\psi^{(1)}(t)\right\rangle=-i(\Omega t)|e\rangle, \\
\left|\psi^{(2)}(t)\right\rangle=-\frac{(\Omega t)^{2}}{2 !}|g\rangle,\left|\psi^{(3)}(t)\right\rangle=i \frac{(\Omega t)^{3}}{3 !}|e\rangle, \ldots .
\end{gathered}
$$

Including the counter-rotating terms, we obtain

$$
\begin{gathered}
\left|\psi^{(0)}(t)\right\rangle=|g\rangle, \\
\left|\psi^{(1)}(t)\right\rangle=\left[\frac{\Omega}{2 \omega}\left(1-e^{2 i \omega t}\right)-i(\Omega t)\right]|e\rangle, \\
\left|\psi^{(2)}(t)\right\rangle=\left[\frac{\Omega^{2}}{4 \omega^{2}}\left(-1+e^{2 i \omega t}\right)\right. \\
\left.-i \frac{\Omega}{2 \omega}(\Omega t) e^{-2 i \omega t}-\frac{(\Omega t)^{2}}{2}\right]|g\rangle, \\
\left|\psi^{(3)}(t)\right\rangle=\left[\frac{\Omega^{3}}{8 \omega^{3}}\left(\frac{5}{2}-e^{-2 i \omega t}-e^{2 i \omega t}-\frac{1}{2} e^{4 i \omega t}\right)\right. \\
+\frac{\Omega^{2}}{4 \omega^{2}}(\Omega t)\left(1-e^{-2 i \omega t}+e^{2 i \omega t}\right) \\
\left.-\frac{\Omega}{4 \omega}(\Omega t)^{2}\left(1-e^{2 i \omega t}\right)+i \frac{(\Omega t)^{3}}{6}\right]|e\rangle, \ldots
\end{gathered}
$$

From these expressions, it is clear that besides the RWA terms (Taylor expansions of $\cos (\Omega t)$ and $\sin (\Omega t)$ ), we have terms proportional to $e^{n(2 i \omega t)}$, multiplied by powers of $\Omega t$. We will discuss in Sec IV A the signatures of these terms in the frequency domain.

An example of the comparison between the exact (numerical) solution of the semiclassical Rabi model and the truncated Picard series is shown in Fig. 1. It is clear that the Picard expansion is suitable only for short times. Indeed, with expansion up to thirty-third order we can faithfully reproduce the exact dynamics only up to less than two Rabi periods. From these plots we can also appreciate small (beyond RWA) oscillations, superposed to the main Rabi oscillations. The amplitude and frequency of these small oscillations will be discussed in Sec IVA.

\section{B. Quantum Rabi model}

In this subsection, we review with more details the Picard expansion for the finite-time quantum Rabi model introduced in Ref. [15]. We expand the state vector in the $\{|l, n\rangle\}$ basis $(l=g, e ; n=0,1,2, \ldots)$ as $|\psi(t)\rangle=$ $\sum_{l, n} C_{l, n}(t)|l, n\rangle$. For every term in the Hamiltonian (5) it is possible to give a diagrammatic representation (see Fig. 2). The interaction vertex is represented by a full circle, a photon by a wavy line, the qubit in the ground (excited) state by a straight line (two parallel straight
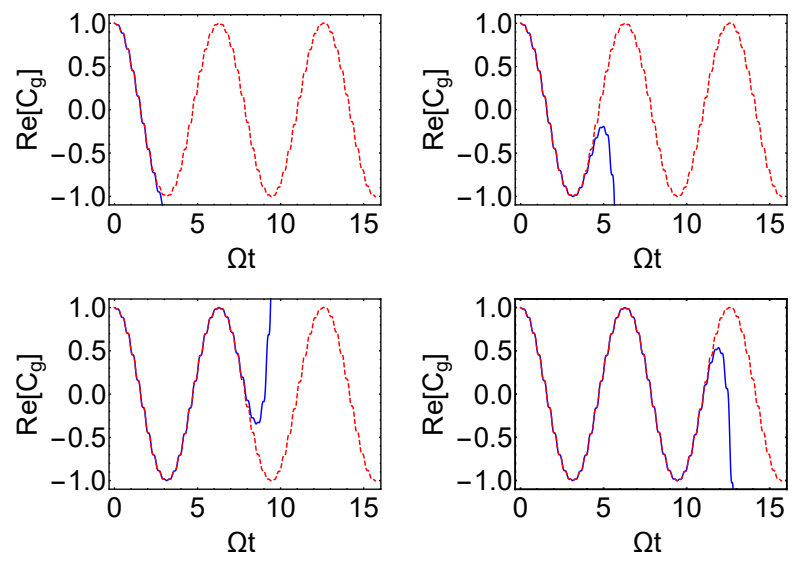

FIG. 1. (Color online) Comparison (for $\operatorname{Re}\left[C_{g}\right]$ ) between the numerical solution of the semiclassical Rabi model (dashed red line) and the Picard series (solid blue line) up to third (top left), eleventh (top right), twenty-first (bottom left), and thirty-third (bottom right) order, for $\Omega / \omega=0.1$.

lines). Time flows from bottom to top. The vertex corresponding to the term proportional to $\sigma_{+} a$ in the Hamiltonian tells us that we start from the qubit in the ground state and a photon. As a consequence of the qubit-field interaction, the photon is absorbed and the qubit is promoted to its excited state. The term $\sigma_{-} a^{\dagger}$ de-excites the atom while creating a photon, $\sigma_{-} a$ simultaneously destroys a photon and de-excites the atom, and $\sigma_{+} a^{\dagger}$ simultaneously creates a photon and excites the atom. The last two terms are responsible of the anti-DCE and DCE effect, respectively.

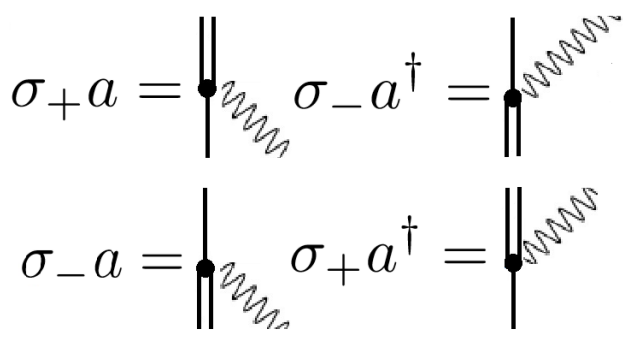

FIG. 2. Vertices associated to the terms in Hamiltonian (5). The vertices in the bottom line correspond to terms neglected within the RWA.

We focus on the initial condition $|\psi(0)\rangle=|g, 0\rangle$, corrsponding to both the qubit and the field in their ground state. Within the RWA, which conserves the total number of excitations $N_{T}=\sigma_{+} \sigma_{-}+a^{\dagger} a$, no excitations are possible and $|\psi(t)\rangle=|g, 0\rangle$ at all times. On the other hand, the dynamics is nontrivial when the terms beyond RWA are included, since one can simultaneously excite the qubit and create a photon, $\sigma_{+} a^{\dagger}|g, 0\rangle=|e, 1\rangle$. The generation of photons from the vacuum is due to the nonadiabatic change of a system parameter (switching of 
the qubit-field coupling constant) and is a manifestation of the (parametric) DCE [7.

To the zeroth-order approximation $\left|\psi^{(0)}(t)\right\rangle=|g, 0\rangle$. Such state is diagrammatically represented as a vertical single line (see the left diagram in Fig. 3), meaning that the qubit remains in its ground state $|g\rangle$, while no photons are emitted. The two horizontal lines in Fig. 3 (left) (as well as in all other diagrams in this paper) mean that interaction is switched on at time $t=0$ (lower line) and switched off at time $t=\tau$ (upper line) That is, these lines outline the fact that we are dealing with finite-time QED.

To compute the first-order terms, we first observe that $H_{I}\left(t^{\prime}\right)\left|\psi^{(0)}\left(t^{\prime}\right)\right\rangle=e^{2 i \omega t^{\prime}} \sigma_{+} a^{\dagger}|g, 0\rangle=e^{2 i \omega t^{\prime}}|e, 1\rangle$. After integrating $H_{I}\left(t^{\prime}\right)\left|\psi^{(0)}\left(t^{\prime}\right)\right\rangle$ from $t^{\prime}=0$ to $t^{\prime}=t$ according to Eq. (9), we obtain

$$
\left|\psi^{(1)}(t)\right\rangle=\frac{\lambda}{2 \omega}\left(1-e^{2 i \omega t}\right)|e, 1\rangle .
$$

The diagrammatic representation of the first-order contribution is shown in Fig. 3 (right): the system starts from the state $|g, 0\rangle$ and performs a transition to the state $|e, 1\rangle$, with the qubit left in the excited state $|e\rangle$ and the emission of a single (real) photon. Note that this diagram is beyond the RWA, since the energy is not conserved: both the qubit and the field start from their ground states and are eventually excited.

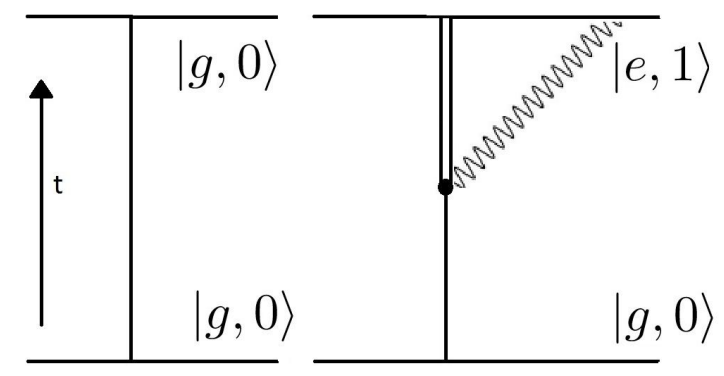

FIG. 3. Diagrammatic representation of the zeroth- (left) and first-order (right) contributions in the Picard series for the quantum Rabi model, with initial condition $|\psi(0)\rangle=|g, 0\rangle$.

To obtain the second-order contributions, we apply $H_{I}\left(t^{\prime}\right)$ to the first-order correction $\left|\psi^{(1)}\left(t^{\prime}\right)\right\rangle$. Since $\left|\psi^{(1)}\left(t^{\prime}\right)\right\rangle \propto|e, 1\rangle$, we obtain terms proportional to $\sigma_{-} a|e, 1\rangle=|g, 0\rangle$ and $\sigma_{-} a^{\dagger}|e, 1\rangle=\sqrt{2}|g, 2\rangle$. These contributions are represented by the diagrams of Fig. 4. Note that in the first case (left diagram) the photon is virtual, while in the second (right diagram) two real photons are emitted. After integrating over time $H_{I}\left(t^{\prime}\right)\left|\psi^{(1)}\left(t^{\prime}\right)\right\rangle$ according to Eq. 10 (with $n=2$ ), we obtain

$$
\begin{gathered}
\left|\psi^{(2)}(t)\right\rangle=i \frac{\lambda^{2}}{2 \omega}\left[t+\frac{i}{2 \omega}\left(1-e^{-2 i \omega t}\right)\right]|g, 0\rangle \\
+i \frac{\sqrt{2} \lambda^{2}}{2 \omega}\left[-t+\frac{i}{2 \omega}\left(1-e^{2 i \omega t}\right)\right]|g, 2\rangle .
\end{gathered}
$$

It is interesting to remark that in the latter term the $\sqrt{2}$ factor is due to the stimulated emission of the second photon by the first one.

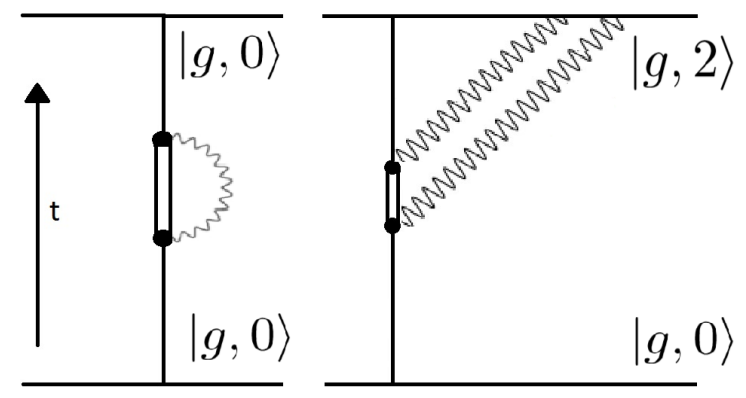

FIG. 4. Same as in Fig. 3, but for the second-order contribtions.

To obtain the third-order contribution, we apply $H_{I}\left(t^{\prime}\right)$ to $\left|\psi^{(2)}\left(t^{\prime}\right)\right\rangle$. As a result, from the term proportional to $|g, 0\rangle$ in $\left|\psi^{(2)}\left(t^{\prime}\right)\right\rangle$ we obtain a term proportional to $|e, 1\rangle$ (top left diagram in Fig. 5, while from the term proportional to $|g, 2\rangle$ we obtain two terms, one proportional to $|e, 3\rangle$ (top right diagram in Fig. 5) and one to $|e, 1\rangle$ (bottom diagram in Fig. 5). After integrating over time $H_{I}\left(t^{\prime}\right)\left|\psi^{(2)}\left(t^{\prime}\right)\right\rangle$, we obtain

$$
\begin{gathered}
\left|\psi^{(3)}(t)\right\rangle=\frac{\lambda^{3}}{4 \omega^{3}}\left[-1+e^{2 i \omega t}-i(\omega t)\left(1+e^{2 i \omega t}\right)\right]|e, 1\rangle \\
+\sqrt{\frac{3}{2}} \frac{\lambda^{3}}{8 \omega^{3}}\left[1-e^{4 i \omega t}+4 i e^{2 i \omega t}(\omega t)\right]|e, 3\rangle \\
+\frac{\lambda^{3}}{4 \omega^{3}}\left[1-e^{2 i \omega t}+2 i(\omega t)-2(\omega t)^{2}\right]|e, 1\rangle
\end{gathered}
$$

where the three terms of this equation correspond, respectively, to the top left, top right, and bottom diagram of Fig. 5). The perturbative treatment outlined in this subsection can be easily iterated to higher orders.

As an example of application of the Picard expansion, we compute the mean number of generated photons, as a function of the qubit-field coupling constant $\lambda$ and of the interaction time $t$. We can see from Fig. 6 that the fourth-order plot (top panel) is in good agreement with the exact solution (bottom panel), provided $\lambda$ and $t$ are not too large.

For $\lambda / \omega=0.1$, the Picard expansion is compared (up to the fourth order) with the exact numerical solution in Fig. 7. It can be seen that the Picard series truncated to the fourth order can reproduce the behavior of $\langle n\rangle$ up to $t / \tau_{s} \approx 0.5$. On the other hand, for this value of $\lambda$ the amplitude and time of the first peak can be estimated already from the first-order expansion. To the first order,

$$
\langle n\rangle(t)=\left(\frac{\lambda}{\omega}\right)^{2} \sin ^{2}(\omega t),
$$

corresponding to the first peak at time $\tau_{p}$, with $\tau_{p} / \tau_{s}=$ $\lambda / \omega$, and peak value $\langle n\rangle\left(\tau_{p}\right)=(\lambda / \omega)^{2}$. As shown in 


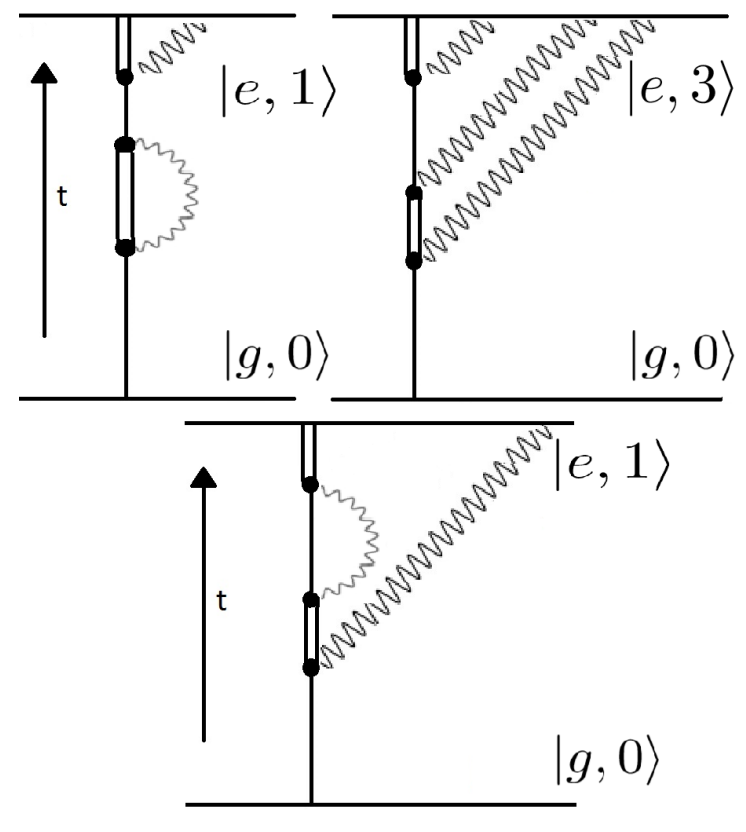

FIG. 5. Same as in Fig. 3. but for the third-order contribtions.

Fig. 8, this analytical prediction for $\tau_{p}$ is in good agreement with the numerical results up to $\lambda / \omega \approx 0.3$.

The oscillations in $\langle n\rangle(t)$, due to the coherent generation (DCE) and destruction (anti-DCE) of photons, are a clear dynamical "smoking gun" of the ultrastrong coupling regime. Such oscillations, as shown in Figs. 6 and 7. are, for relatively small values of $\lambda / \omega$, regular. At small times, a quasi-periodic behavior with frequency $2 \omega$ is clearly seen, and also predicted by first-order perturbation theory, Eq. 16). This reasult might be interesting for experimental investigations in that clear features of the DCE are observable with short interaction times and relatively small interaction strengths.

\section{MAGNUS EXPANSION}

The Magnus expansion starts by assuming that an exponential form for the (unitary) time-evolution operator $U(t)$ (defined by $|\psi(t)\rangle=U(t)|\psi(0)\rangle$ ) exists:

$$
U(t)=e^{\Omega(t)}, \quad \Omega(0)=0,
$$

with a series expansion for $\Omega$ :

$$
\Omega(t)=\sum_{n=1}^{\infty} \Omega^{(n)}(t) .
$$

An approximate expression for the time-evolution operator is obtained by truncation of the Magnus expansion.
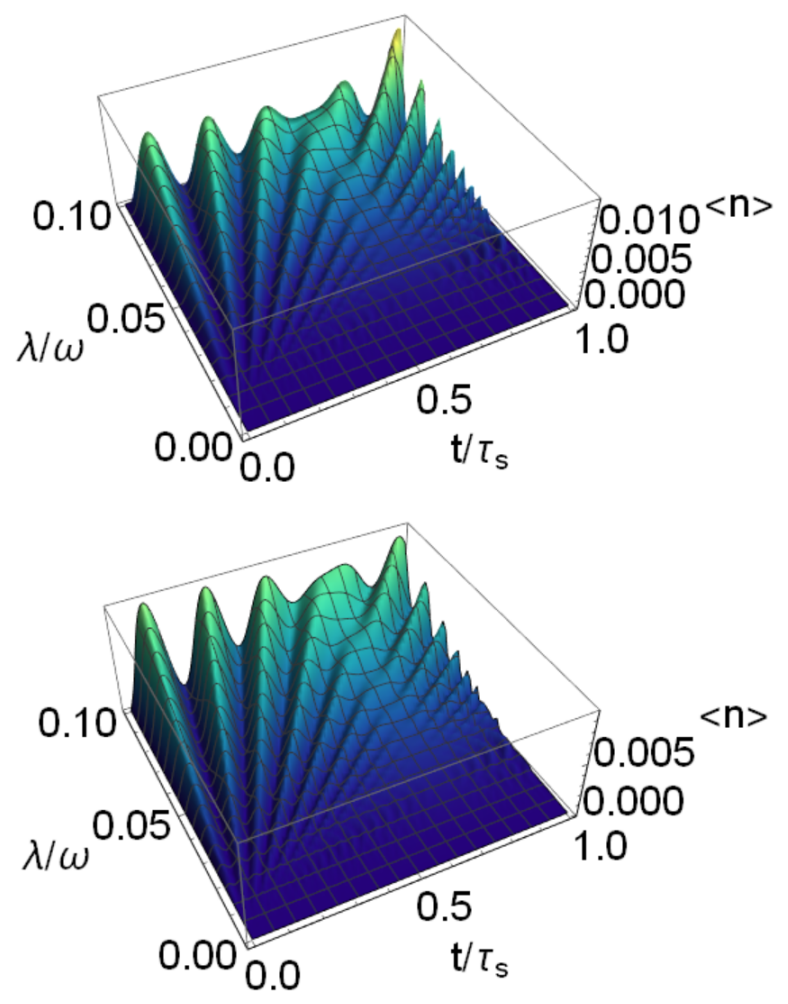

FIG. 6. (Color online) Mean number of generated photons $\langle n\rangle$ as a function of the coupling strength $\lambda$ (in units of $\omega$ ) and of the interaction time $t$, measured in units of the swapping time $\tau_{s}=\pi / 2 \lambda$. The fourth-order Picard expansion (top) is compared with the numerical results (bottom).

The first few terms in such expansion are

$$
\begin{gathered}
\Omega^{(1)}(t)=\int_{0}^{t} d t_{1} A\left(t_{1}\right), \\
\Omega^{(2)}(t)=\frac{1}{2} \int_{0}^{t} d t_{1} \int_{0}^{t_{1}} d t_{2}\left[A\left(t_{1}\right), A\left(t_{2}\right)\right], \\
\Omega^{(3)}(t)=\frac{1}{6} \int_{0}^{t} d t_{1} \int_{0}^{t_{1}} d t_{2} \int_{0}^{t_{2}} d t_{3} \\
\left(\left[A\left(t_{1}\right),\left[A\left(t_{2}\right), A\left(t_{3}\right)\right]\right]+\left[A\left(t_{3}\right),\left[A\left(t_{2}\right), A\left(t_{1}\right)\right]\right]\right),
\end{gathered}
$$

where we have defined the (anti-Hermitian) operator $A(t)=-i H_{I}(t)$, with $H_{I}$ Hamiltomian in the interaction picture. For a derivation of the terms $\Omega^{(n)}(t)$ see, e.g., Ref. 26]. Note that, since the expansion is for $\Omega$ and not for $U$ as in the Picard series, the Magnus expansion provides a unitary perturbation theory, in contrast to the Picard series. This is one of the most appealing features of the Magnus expansion. The Magnus expansion, in particular conditions for the convergence of the Magnus series and several applications of the method, including its use as a numerical integrator, are reviewed in Ref. 26]. Hereafter, we shall discuss applications of the Magnus expansion to the Rabi model. 

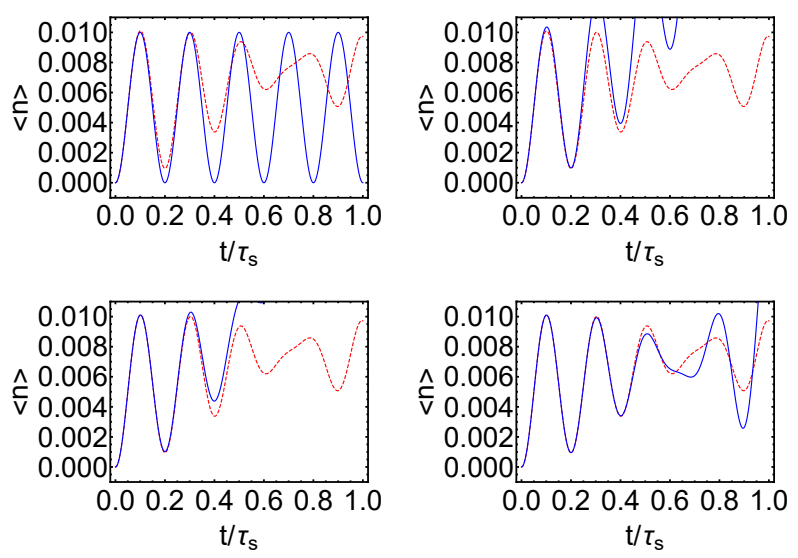

FIG. 7. (Color online) Mean number of photons as a function of time, with the numerical results (dashed red line) compared with the Picard expansion (solid blue line) truncated to the first (top left), second (top right), third (bottom left), and fourth (bottom right) order, for $\lambda / \omega=0.1$.

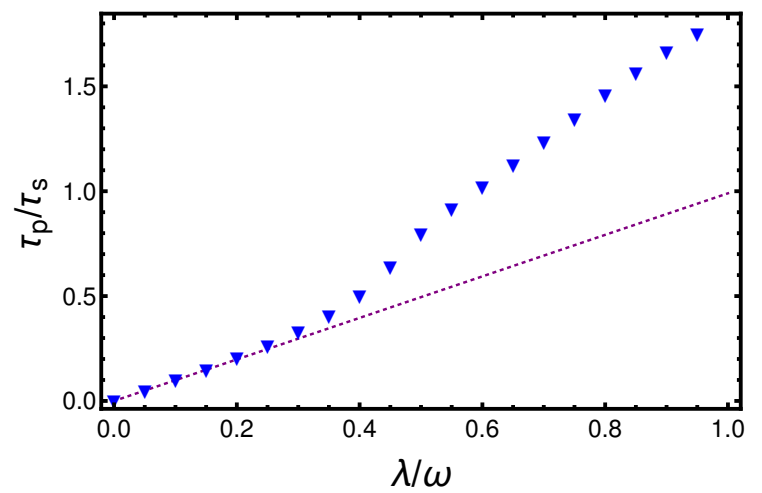

FIG. 8. (Color online) Time $\tau_{p}$ of the first peak in $\langle n\rangle(t)$ : comparison of the first-order (dashed line) with the numerical results (triangles).

\section{A. Semiclassical Rabi model}

We write explicitly the first three terms of the Magnus expansion for the semiclassical Rabi model. Let $\Omega_{i j}^{(n)}=$ $\left\langle i\left|\Omega^{(n)}\right| j\right\rangle$, with $i, j=g, e$, denote the matrix elements of $\Omega^{(n)}$ in the $\{|g\rangle,|e\rangle\}$ basis. From Eq. 19 , using the semiclassical Rabi Hamiltonian (4) we obtain

$$
\begin{gathered}
\Omega_{g g}^{(1)}(t)=\Omega_{e e}^{(1)}(t)=0 \\
\Omega_{g e}^{(1)}(t)=-\frac{\Omega}{2 \omega}\left(1-e^{-2 i \omega t}+2 i \omega t\right)=-\left[\Omega_{e g}^{(1)}(t)\right]^{\star} \\
\Omega_{g g}^{(2)}(t)=\Omega_{e g}^{(2)}(t)=0 \\
\frac{i \Omega^{2}}{4 \omega^{2}}(-2 \omega t \cos (2 \omega t)+\sin (2 \omega t)) \\
=-\left[\Omega_{e e}^{(2)}(t)\right]
\end{gathered}
$$

$$
\begin{gathered}
\Omega_{g g}^{(3)}(t)=\Omega_{e e}^{(3)}(t)=0 \\
\Omega_{g e}^{(3)}(t)=\frac{\Omega^{3}}{8 \omega^{3}}\left[-3+i \omega t+\frac{4}{3} \omega^{2} t^{2}\right. \\
+\left(\frac{3}{2}+2 i \omega t-\frac{2}{3} \omega^{2} t^{2}\right) e^{-2 i \omega t} \\
+\left(\frac{7}{6}-\frac{4}{3} i \omega t-\frac{2}{3} \omega^{2} t^{2}\right) e^{2 i \omega t} \\
\left.+\left(\frac{1}{3}+\frac{1}{3} i \omega t\right) e^{-4 i \omega t}\right]=-\left[\Omega_{e g}^{(3)}(t)\right]^{\star}
\end{gathered}
$$

Within the RWA, the semiclassical Rabi model is, in the interaction picture, time-independent, and therefore the Magnus expansion reduces to its first-order term, $\Omega(t)=\Omega^{(1)}(t)=-i H_{I} t$. On the other hand, when the terms beyond RWA are taken into account, in general $\left[A\left(t_{1}\right), A\left(t_{2}\right)\right] \neq 0$ if $t_{1} \neq t_{2}$ and therefore we must consider also higher-order terms in the Magnus expansion.

As an example, in Fig. 9 (left panel) we compare the Magnus expansion, truncated to the fourth order, with the numerical integration of the Schrödinger equation via a fourth-order Runge-Kutta method. If we compare these results with those obtained by means of the Picard series (see Fig. 1), it is clear that the Magnus expansion allows us to address much longer evolution times already at small orders.

On the other hand, the convergence of the Magnus expansion is not guaranteed at all times. More precisely, a sufficiently condition [27, 28, for the convergence of the Magnus expansion is that

$$
\int_{0}^{t}\left\|A\left(t^{\prime}\right)\right\|_{2} d t^{\prime}<\pi
$$

where $\|A\|_{2}$ is the square root of the largest eigenvalue of $A^{\dagger} A$. In the example of Fig. 9, this criterion ensures convergence for times $t<t_{c}$, with $\Omega t_{c} \approx 5.1$ (vertical dashed line in the figure). For $t>t_{c}$, the strong oscillations and the discrepancy between the Magnus expansion truncated to the fourth-order and the exact numerical solution, suggest a different numerical approach. That is, we concatenate truncated Magnus expansions. With this approach, we can address arbitrarily long time scales. For instance, Fig. 9 (right panel) shows the good agreeement between the numerical solution and the concatenation of five first-order Magnus expansions.

To further assess the validity of the Magnus expansion, we follow the dynamics up to 30 Rabi periods $(\Omega t=60 \pi)$, by concatenating $\mathcal{N}$ times the fourth-order Magnus expansion, and then compute the Fourier transforms $F$ of $C_{g}$ and $C_{e}$. As an example, we show in Fig. $10 F\left[\operatorname{Re}\left(C_{g}\right)\right]$, for different values of $\mathcal{N}$. We can see that $\mathcal{N}=3 \times 10^{3}$ allow us to reproduce the main features of the Fourier spectrum: for that purpose, more than $10^{5}$ time steps are necessary when using the Runge-Kutta method (see the bottom right panel of Fig. 10). The Magnus expansion can then be used as a numerical integrator, more efficient for this problem than the Runge-Kutta mehod, as it allows much longer time steps. 

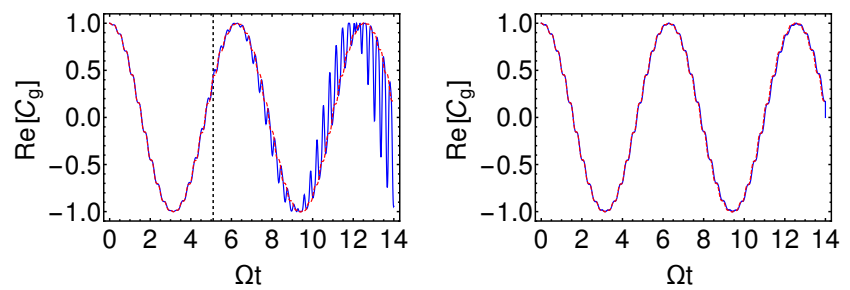

FIG. 9. (Color online) Comparison (for $\operatorname{Re}\left[C_{g}\right]$ ) between the numerical solution of the semiclassical Rabi model (dashed red line) and the Magnus expansion (solid blue line), up to fourth order (left) or iterating five times the first order expansion (right), for $\Omega / \omega=0.1$. The dashed line shows the time $(\Omega t \approx 5.1)$ up to which convergence of the Magnus expansion is guaranteed by criterion 23.
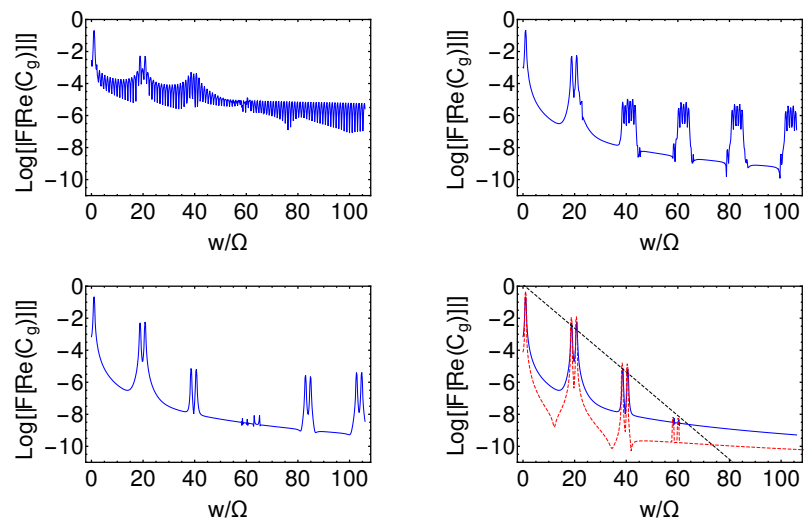

FIG. 10. (Color online) Fourier transform $F$ of $\operatorname{Re}\left(C_{g}\right)$ (arbitrary units in the plot), obtained from integration of the semiclassical Rabi model up to $\Omega t=60 \pi$, with $\Omega / \omega=0.1$, iterating the fourth-order Magnus expansion $\mathcal{N}=5$ (top left), 100 (top right), 500 (bottom left), and 3000 (bottom right) times. The dashed red curve in the bottom right panel is instead obtained by fourth-order Runge-Kutta integration of the equations of motion, with $5.12 \times 10^{5}$ points. The dashed line $\log \left[\left|F\left[\operatorname{Re}\left(C_{g}\right)\right]\right|\right]=a-b(w / \Omega)$, with $a \approx 0.106$ and $b \approx 0.136$, fits the decay of the peaks in the Fourier transform.

The Fourier spectrum has characteristic double-peaks. More precisely, Fig. 10 exhibits a single peak at the Rabi frequency $\Omega$, and doublets at frequencies $2 n \omega \pm \Omega$, with $n=1,2,3, \ldots$. Such features can be qualitatively explained as follows. The peak at frequency $\Omega$ corresponds to Rabi oscillations and already exists within the RWA. On the other hand, the doublets are structures beyond RWA, which can be conveniently understood from the Picard series. At each order of the Picard expansion, we integrate in time terms proportional to $e^{ \pm 2 i \omega t}$ times the wave-function at the previous order. We therefore generate new harmonics at higher frequency as we increase the perturbative order in the Picard series. Terms proportional to $e^{ \pm 2 i n \omega t}$ multiply the Rabi oscillations, proportional to $e^{i \Omega t}$, and therefore in conclusion we generate harmonics at frequencies $2 n \omega \pm \Omega$. Note that each inte- gration in time of $e^{ \pm 2 i \omega t}$ implies a decay of the weight of the corresponding harmonic by a factor $1 /(2 \omega)$. If we write the Schrödinger equation for the semiclassical Rabi model 4 as

$$
\left[\begin{array}{c}
\dot{C}_{g}(t) \\
\dot{C}_{e}(t)
\end{array}\right]=-i f(t)\left[\begin{array}{cc}
0 & 1+e^{-2 i \omega t} \\
1+e^{2 i \omega t} & 0
\end{array}\right]\left[\begin{array}{l}
C_{g}(t) \\
C_{e}(t)
\end{array}\right]
$$

we can clearly see that at each order of the Picard series we improve the approximation for either $C_{g}$ or $C_{e}$. Therefore, we need two steps in the Picard expansion to improve $C_{g}$ (or $C_{e}$ ) and generate new harmonics. Since this implies two integrations in time, the harmonics at frequencies $2 n \omega \pm \Omega$ are scaled by a factor $[\Omega /(2 \omega)]^{2}$ with respect to the harmonics at frequencies $2(n-1) \omega \pm \Omega$. This estimate is in good agreement with the numerical results of Fig. 10 . Indeed, for $\Omega / \omega=0.1$ the decay of the first peaks in the Fourier transform is fitted by an exponential law, $\log \left[\left|F\left[\operatorname{Re}\left(C_{g}\right)\right]\right|\right]=a-b(w / \Omega)$, with $a \approx 0.106$ and $b \approx 0.136$. This implies that the ratio between the amplitude of nearby doublets is approximately equal to $10^{-2(\omega / \Omega) b} \approx 1 / 525$, not far from $[\Omega /(2 \omega)]^{2}=1 / 400$. A more precise calculation appears difficult, since at each perturbative order new harmonics are generated but also the weight of the already existing harmonics is modified. Note that in the Magnus series, since we have an exponential approximation theory (i.e., we consider $e^{\Omega}$, with a truncated expansion for $\Omega$ ), higher-order harmonics are visible already at the lowest orders.

The above discussion can be visualized by means of the analog circuit reported in Fig. 11. It corresponds to two orders in the Picard expansion, and each integration brings a factor $\Omega /(2 \omega)$. The signal $\left(C_{g}\right.$ and $\left.C_{e}\right)$ can be reinjected and at each loop the approximation is improved, adding each time two more orders in the Picard series.

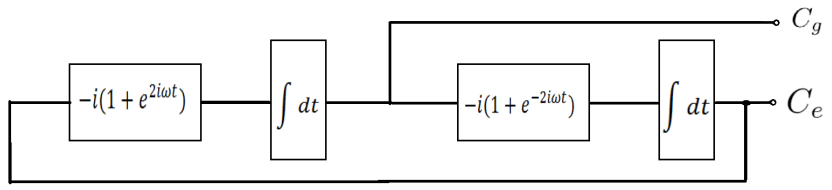

FIG. 11. Schematic drawing of an analog circuit for the integration of the Schrödinger equation (24) for the semiclassical Rabi model.

Finally, we point out that there is an interesting analogy, in particular with respect to the occurrence of doublets, between the semiclassical Rabi model and the Mathieu equation in an appropriate range of parameters, see Appendix A.

\section{B. Quantum Rabi model}

The Magnus expansion can also be applied to the quantum Rabi model, using Eqs. (17), (18) and (19). For the 
sake of simplicity, we do not report explicit expressions for $\Omega^{(n)}(t)$. As the Hilbert space is infinite-dimensional, we cannot use convergence criteria like Eq. (23), since the eigenvalues of $A^{\dagger} A$ are not upper bounded. On the other hand, for any given initial condition the Hilbert space actually explored by the dynamics is finite. For instance, if initially both the field and the qubit are prepared in their ground state, as discussed in Sec. IIIB the mean number of photons does not grow indefinitely but oscillates due to coherent generation (DCE) and destruction (anti-DCE) of photons. Hence, we expect convergence of the Magnus expansion for sufficiently short integration times. Such expectation is borne out by numerical data, as shown in Fig. 12.
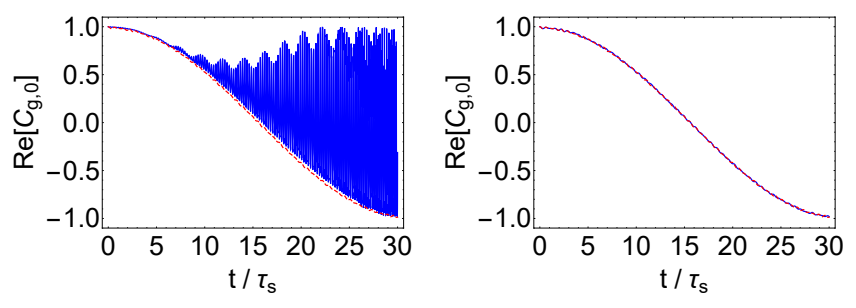

FIG. 12. (Color online) Comparison (for $\operatorname{Re}\left[C_{0 g}\right]$ ) between the numerical solution of the semiclassical Rabi model (dashed red line) and the Magnus expansion (solid blue line), up to fourth order (left) or iterating a hundred times the fourth order expansion (right), for $\lambda / \omega=0.12$. Note that the initial condition we used, $C_{g, 0}(t=0)=1$, is such that within the RWA the dynamics is trivial, $C_{g, 0}(t)=1$ at all times.

\section{CONCLUSIONS}

In this paper, we have applied the Picard and Magnus expansions to the ultrastrong matter-field coupling, in the paradigmatic Rabi model. The Picard series, truncated to low orders, is suitable only for short interaction times. On the other hand, we have shown that the Magnus expansion, through concatenation, is an efficient numerical integrator, in that it allows time steps much longer than in the Runge-Kutta method.

We have highlighted clear features of the dynamics in the ultrastrong coupling regime, and in particular of the dynamical Casimir effect. Regular oscillations in the mean number $\langle n\rangle$ of photons, due to the coherent generation (DCE) and destruction (anti-DCE) of photons take place. This reasult provides a clear "smoking gun" of the DCE, which might be of interest for experimental investigations in circuit $\mathrm{QED}$, in that the above oscillations are observable with short interaction times and relatively small interaction strengths.

We have shown that the Fourier spectrum of motion in the semiclassical Rabi model exhibits a peak at the Rabi frequency $\Omega$ and doublets at frequencies $2 n \omega \pm \Omega$, with $n$ positive integer. While the Rabi frequency is trivially obtained by solving the Rabi model within the rotating wave approximation, the doublets are features beyond the RWA. Both the oscillations in $\langle n\rangle$ and the doublets can be explained by means of the Picard series. The Fourier analysis can be extended also to the quantum Rabi model finding similar, even though more complicated structures with doublets. Finally, the analogy with the Mathieu equation highligths the fact that doublets are a general feature of time-modulated systems.

Acknowledgments: We acknowledge support by the INFN through the project "QUANTUM".

\section{Appendix A: Analogy between the semiclassical Rabi model and the Mathieu equation}

Let us consider the Mathieu equation [29]

$$
\ddot{y}(t)+[a-2 q \cos (\omega t)] y(t)=0,
$$

with $a$ and $q$ real constants 30 . If we define $p \equiv \dot{y}$, we can write the Mathieu equation as

$$
\left[\begin{array}{l}
\dot{y}(t) \\
\dot{p}(t)
\end{array}\right]=\left[\begin{array}{cc}
0 & 1 \\
-[a-2 q \cos (2 t)] & 0
\end{array}\right]\left[\begin{array}{l}
y(t) \\
p(t)
\end{array}\right] .
$$

Similarities and differences between this equation and the Schrödinger equation (24) for the semiclassical Rabi model are self-evident. This point can also be visualized by comparing the analog circuit of Fig. 13 for the Mathieu equation with the circuit of Fig 11 for the semiclassical Rabi model.

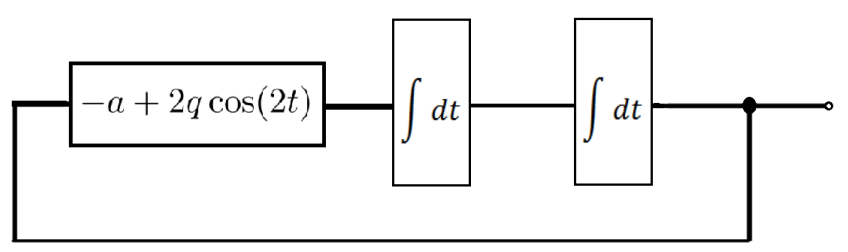

FIG. 13. Schematic drawing of an analog circuit for the integration of the Mathieu equation.

As for the semiclassical Rabi model, there are doublets in the Fourier spectrum, see Fig. 14. Such doublets are generated by time-dependent terms, that is, in the Rabi model the terms beyond RWA, and in the Mathieu equation the term $2 q \cos (\omega t)$, which corresponds to a frequency modulation. For the Rabi model the evolution is unitary, while this is not the case for the Mathieu equation. However, this lack of unitarity does not affect in any way the reason why doublets are present. On the other hand, we shall limit ourselves to the stable region with small values of the parameters $a$ and $q$, since for large values of these parameters there are strong nonlinearities and a more complicated treatment is needed. We can see from Fig 14 that in the Fourier transform $[F y](w)$ of $y(t)$ there is a single peak at $w=\sqrt{a}$ and doublets at frequencies $n \omega \pm \sqrt{a}$, with $n=1,2,3, \ldots$. Similarly to the semiclassical Rabi model, this non-trivial structure may be explained on the basis of the Picard expansion. 


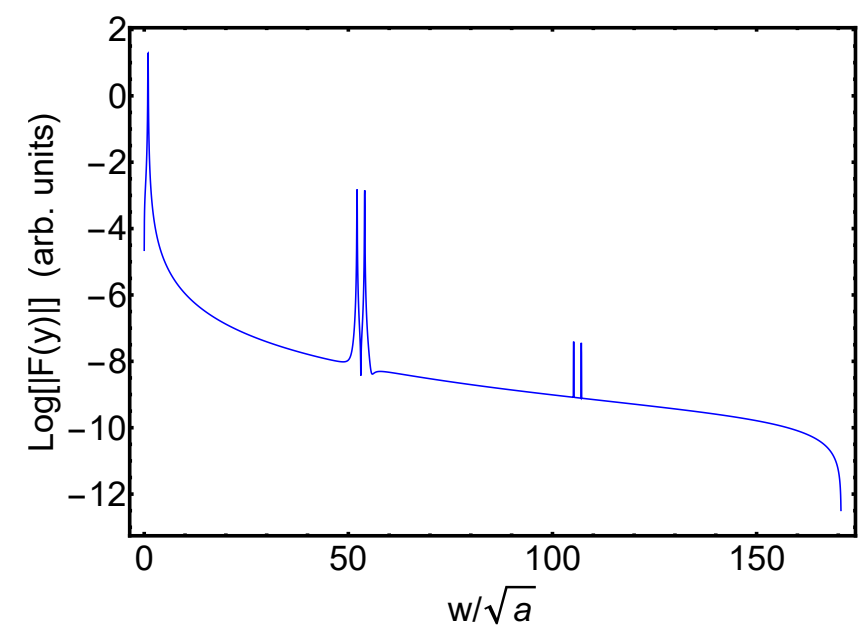

FIG. 14. Fourier transform $[F y](w)$ of the solution $y(t)$ of the Mathieu equation A1 with initial conditions $y(t=0)=1$, $p(t=0)=0$, integrated up to $t=200$. Parameter values: $a=0.5, q=0.1$, and $\omega=40$.

[1] J. Bourassa, J. M. Gambetta, A. A. Abdumalikov, Jr., O. Astafiev, Y. Nakamura, and A. Blais, Phys. Rev. A 80, 032109 (2009).

[2] T. Niemczyk, F. Deppe, H. Huebl, E. Menzel, F. Hocke, M. J. Schwarz, J. J. García-Ripoll, D. Zueco, T. Hümmer, E. Solano, A. Marx, and R. Gross, Nature Phys. 6, 772 (2010).

[3] P. Forn-Díaz, J. Lisenfeld, D. Marcos, J. J. García-Ripoll, E. Solano, C. J. P. M. Harmans, and J. E. Mooij, Phys. Rev. Lett. 105, 237001 (2010).

[4] P. Forn-Díaz, J. J. García-Ripoll, B. Peropadre, J.-L. Orgiazzi, M. A. Yurtalan, R. Belyansky, C. M. Wilson, and A. Lupascu, Nature Phys. 13, 39 (2017).

[5] F. Yoshihara, T. Fuse, S. Ashhab, K. Kakuyanagi, S. Saito, and K. Semba, Nature Phys. 13, 44 (2017).

[6] G. T. Moore, J. Math. Phys. (N.Y.) 11, 2679 (1970).

[7] V. V. Dodonov, Phys. Scripta 82, 038105 (2010).

[8] P. D. Nation, J. R. Johansson, M. P. Blencowe, and F. Nori, Rev. Mod. Phys. 84, 1 (2012).

[9] J.-C. Jaskula, G. B. Partridge, M. Bonneau, R. Lopes, J. Ruaudel, D. Boiron, and C. I. Westbrook, Phys. Rev. Lett. 109, 220401 (2012).

[10] S. Koghee and M. Wouters, Phys. Rev. Lett. 112, 036406 (2014).

[11] S. Felicetti, M. Sanz, L. Lamata, G. Romero, G. Johansson, P. Delsing, and E. Solano, Phys. Rev. Lett. 113, 093602 (2014).

[12] C. Sabín, I. Fuentes, and G. Johansson, Phys. Rev. A 92, 012314 (2015).

[13] C. Sabín and 'G. Adesso, Phys. Rev. A 92, 042107 (2015).

[14] R. Stassi, S. De Liberato, L. Garziano, B. Spagnolo, and S. Savasta, Phys. Rev. A 92, 013830 (2015).

[15] G. Benenti, S. Siccardi, and G. Strini, Eur. Phys. J. D 68, 139 (2014).

[16] G. Benenti, A. D'Arrigo, S. Siccardi, and G. Strini, Phys.
Rev. A 90, 052313 (2014).

[17] G. Benenti and G. Strini, Phys. Rev. A 91, 020502(R) (2015).

[18] F. Hoeb, F. Angaroni, J. Zoller, T. Calarco, G. Strini, S. Montangero, and G. Benenti, Phys. Rev. A 96, 033851 (2017).

[19] C. M. Wilson, G. Johansson, A. Pourkabirian, M. Simoen, J. R. Johansson, T. Duty, F. Nori, and P. Delsing, Nature (London) 479, 376 (2011).

[20] P. Lähteenmäki, G. S. Paraoanu, J. Hassel, and P. J. Hakonen, PNAS 110, 4234 (2013).

[21] K. Nomoto and R. Fukuda, Progr. Theor. Phys. 86, 269 (1991).

[22] P. Meystre and M. Sargent III, Elements of quantum optics (4th Ed.) (Springer-Verlag, Berlin, 2007).

[23] For a recent collection of articles on semiclassical and quantum Rabi models, see D. Braak, Q.-H. Chen, M. T Batchelor, and E. Solano, J. Phys. A: Math. Theor. 49, 300301 (2016).

[24] I. M. de Sousa and A. V. Dodonov, J. Phys. A: Math. Theor. 48, 245302 (2015); D. S. Veloso and A. V. Dodonov, J. Phys. B: At. Mol. Opt. Phys. 48, 165503 (2015).

[25] A. Motazedifard, M. H. Naderi, and R. Roknizadeh, J. Opt. Soc. Am. B 32, 1555 (2015).

[26] S. Blanes, F. Casas, J. A. Oteo, and J. Ros, Phys. Rep. 470, 151 (2009).

[27] P. C. Moan and J. Niesen, J. Found. Comp. Math. 8, 291 (2008).

[28] F. Casas, J. Phys. A 40, 15001 (2007).

[29] N. W. McLachlan, Theory and Applications of Mathieu Functions (Oxford University Press, 1947).

[30] We use a parametrization of the Mathieu equation which makes it easier to compare with the semiclassical Rabi model. The standard Mathieu equation instead reads [29]

$$
\ddot{y}(t)+[a-2 q \cos (2 t)] y(t)=0 .
$$

